\title{
Modeling Ullage Dynamics of Tank Pressure Control Experiment during Jet Mixing in Microgravity
}

\author{
O. Kartuzova and M. Kassemi \\ National Center for Space Exploration Research (NCSER) \\ NASA Glenn Research Center \\ Cleveland, $\mathrm{OH} 44135$
}

\begin{abstract}
A CFD model for simulating the fluid dynamics of the jet induced mixing process is utilized in this paper to model the pressure control portion of the Tank Pressure Control Experiment (TPCE) in microgravity ${ }^{1}$. The Volume of Fluid (VOF) method is used for modeling the dynamics of the interface during mixing. The simulations were performed at a range of jet Weber numbers from non-penetrating to fully penetrating. Two different initial ullage positions were considered. The computational results for the jet-ullage interaction are compared with still images from the video of the experiment. A qualitative comparison shows that the CFD model was able to capture the main features of the interfacial dynamics, as well as the jet penetration of the ullage.
\end{abstract}
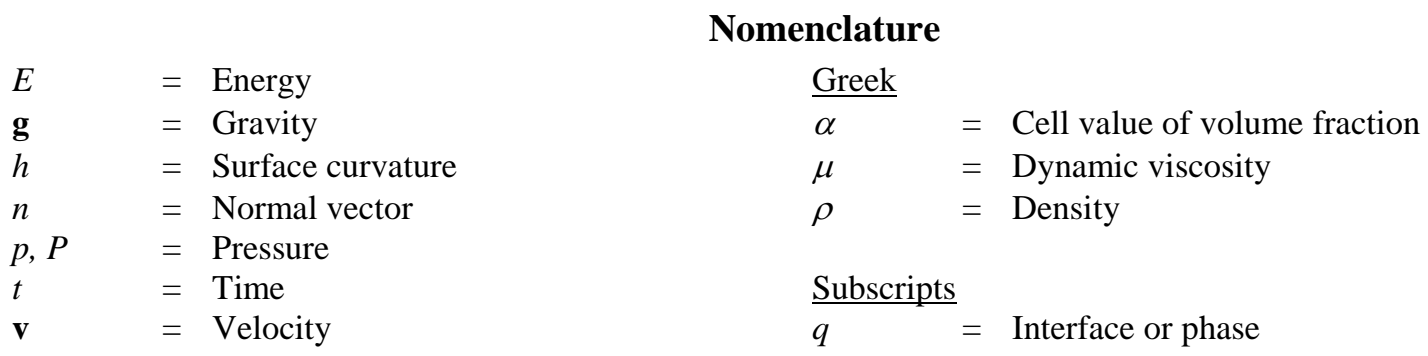

\section{Introduction}

The cost of development and the risk of operations for future NASA exploration missions employing inspace cryogenic storage and transfer systems can be significantly reduced by improving the capability of the Computational Fluid Dynamics (CFD) models that provide predictive simulations of the two-phase fluid dynamics and heat transfer phenomena that govern the performance of the propellant and life support systems in settled and unsettled states. The development and validation of the CFD models against both full scale cryogenic ground-based experiments (settled conditions) and subscale micro-g flight data (unsettled conditions) forms an integral part of the NASA Evolvable Cryogenics project (eCryo). In order to address this need of the eCryo project, the goal of the present paper is to assess the ability of the current two-phase CFD models to predict the fluid dynamics of jet-induced mixing in microgravity.

The data provided by the Tank Pressure Control Experiment (TPCE) were selected for model validation in this study. This experiment flew on the Space Shuttle in 1991 (STS-43). The objectives of the TPCE included characterizing the fluid and interface dynamics during jet mixing in microgravity, and providing data for use in the validation of CFD models of storage tanks under unsettled conditions ${ }^{1}$. The experiment utilized a small scale transparent cylindrical tank, partially filled with a simulant fluid (Freon-113). Two heaters were installed inside the tank for the tank self-pressurization experiments, one at the top of the dome opposite the nozzle and one at the center of the tank near the wall. A pump drew liquid out of the tank through a liquid acquisition device (LAD) and returned it via an axial jet during the tank pressure control tests. Video recording of the tank was conducted in order to capture the ullage dynamics; however, due to data storage limitations, only the first 2 minutes of each tank self-pressurization test and the first 4 minutes of each jet mixing test were recorded. Further details of this experiment can be found in NASA report NASA-CR-191012 (1993) by Michael Bentz ${ }^{1}$.

Previous CFD work related to the TCPE were performed by Wendl et al. ${ }^{2}$ and Breisacher and Moder ${ }^{3}$. Wendl et al. ${ }^{2}$ examined the formation of geysers by an axial jet using a 2D CFD code prior to the experiment's operation. Breisacher and Moder studied the ullage dynamics that occurred during the TPCE pressure control experiments utilizing the Flow3D CFD code ${ }^{3}$. In the latter study, the jet penetration of the ullage was correctly captured by the

American Institute of Aeronautics and Astronautics 
model, and the ullage shapes and dynamics, as well as the final equilibrium position of the ullage, were qualitatively reproduced ${ }^{3}$.

The main focus of the present work is to model the jet mixing segments of TPCE test runs 11, 13, 15 and 4 using the Volume of Fluid (VOF) model of the ANSYS Fluent CFD code, and to validate the two phase CFD model against the experimental results. The details of the test runs considered for model validation are summarized in Table 1.

Table 1: Details of computational cases

\begin{tabular}{cccc}
\hline $\begin{array}{c}\text { Test } \\
\text { Run }\end{array}$ & $\begin{array}{c}\text { Weber } \\
\text { Number }\end{array}$ & $\begin{array}{c}\text { Flow rate, } \\
\text { l/min }\end{array}$ & $\begin{array}{c}\text { Jet Velocity, } \\
\mathbf{m} / \mathbf{s}\end{array}$ \\
\hline 11 & 0.71 & 0.59 & 0.1219 \\
15 & 4.74 & 1.53 & 0.3150 \\
13 & 15.55 & 2.78 & 0.5709 \\
4 & 4.74 & 1.53 & 0.3150 \\
\hline
\end{tabular}

\section{Mathematical Model}

\section{A. Governing Equations}

The fluid flow and heat transfer in the tank are described in terms of the continuity, Navier-Stokes, and energy equations for both phases:

$$
\begin{gathered}
\frac{\partial \rho}{\partial t}+\nabla(\rho \mathbf{v})=0 \\
\frac{\partial}{\partial t}(\rho \mathbf{v})+\nabla(\rho \mathbf{v v})=-\nabla p+\nabla\left[\mu_{e f f}\left(\nabla \mathbf{v}+\nabla v^{T}\right)\right]+\rho \mathbf{g}+\mathbf{F}_{v o l}, \\
\frac{\partial}{\partial t}(\rho E)+\nabla(\mathbf{v}(\rho E+p))=\nabla\left(k_{e f f} \nabla T\right)+S_{h} .
\end{gathered}
$$

In the present study, the liquid phase is treated as incompressible. The vapor is modeled as a compressible ideal gas. All of the thermophysical and thermodynamic properties of the fluids are taken from the NIST Chemistry WebBook ${ }^{4}$ at saturation conditions.

The primary focus of this study is to capture the main dynamics of the fluid flow and jet-ullage interactions. Therefore, although the energy equation was solved, all of the walls were treated as adiabatic, and any phase change mass transfer between the liquid and vapor phases was not considered. The movement of the interface is captured diffusely using the Volume of Fluid (VOF) method, as promulgated by Hirt and Nichols ${ }^{5}$; this method is described below.

\section{B. VOF Model}

In the VOF method, a volume fraction is defined in each cell such that the volume fractions of all of the phases sum to unity. In each cell, the change in the interface can be tracked by solving a continuity equation for the volume fraction of the $q^{\text {th }}$ phase:

$$
\frac{1}{\rho_{q}}\left[\frac{\partial}{\partial t}\left(\alpha_{q} \rho_{q}\right)+\nabla \cdot\left(\alpha_{q} \rho_{q} \mathbf{v}_{q}\right)=S_{\alpha_{q}}\right]
$$

where the volume fraction for the primary phase is determined from:

$$
\sum_{q=1}^{n} \alpha_{q}=1
$$

In the VOF method, the field variables and properties are defined in terms of the volume fraction, which for a general system can be written as:

$$
\rho=\sum_{q=1}^{n} \alpha_{q} \rho_{q}, \quad \mu_{e f f}=\sum_{q=1}^{n} \alpha_{q} \mu_{e f f_{q^{\prime}}} \quad k_{e f f}=\sum_{q=1}^{n} \alpha_{q} k_{e f f_{q}} .
$$

In this fashion, the continuity, momentum, and energy equations, as described by Eq. (1) - (3), can be solved throughout the domain for the temperatures and velocities in the two phases. In the VOF model, the energy $(E)$ and temperature $(T)$ are treated as mass-averaged variables:

$$
E=\frac{\sum_{q=1}^{n} \alpha_{q} \rho_{q} E_{q}}{\sum_{q=1}^{n} \alpha_{q} \rho_{q}}
$$

American Institute of Aeronautics and Astronautics 
where $E_{q}$ is based on the specific heat of the $q^{\text {th }}$ phase and the shared temperature.

In the present implementation, the surface tension forces at the interface are modeled via the Continuum Surface Force (CSF) model of Brackbill et al. ${ }^{6}$. In this model, the surface tension forces at the interface are transformed into a volume force $\left(\mathbf{F}_{v o l}\right)$, which is added as a source to the momentum equation:

$$
\mathbf{F}_{\text {vol }}=\sum_{\text {pairs } i j, i<j} \sigma_{i j} \frac{\alpha_{i} \rho_{i} h_{i} \nabla \alpha_{j}+\alpha_{j} \rho_{j} h_{j} \nabla \alpha_{i}}{\frac{1}{2}\left(\rho_{i}+\rho_{j}\right)},
$$

where $h_{i}$ is the surface curvature calculated from the local gradients in the surface normal at the interface:

$$
h_{i}=\nabla \cdot \widehat{\mathbf{n}} \text {. }
$$

\section{Turbulence modeling}

The turbulence in the tank is modeled by utilizing the Shear Stress Transport $k$ - $\omega$ model of Menter ${ }^{7}$. This model is similar to the standard $k-\omega$ model of $\mathrm{Wilcox}^{8}$, but has the ability to account for the transport of the principal shear stress in adverse pressure gradient boundary layers. The model is based on the assumption of Bradshaw et al. ${ }^{9}$ that the principal shear stress is proportional to the turbulent kinetic energy, which is introduced into the definition of the eddy-viscosity. These features make the kw-SST model more accurate and reliable for a wider class of flows than the standard $k$ - $\omega$ model $^{10}$. In the VOF model, continuity of the turbulent quantities is inherently assumed since one set of equations for the turbulent kinetic energy and dissipation rate is solved for both phases throughout the domain, with the fluid properties varying according to the local volume fraction value.

\section{Numerical Implementation}

The TPCE tank geometry consists of a cylindrical section with a $0.254 \mathrm{~m}$ diameter and two hemispherical domes. The tank is filled with Freon r-113 to about 83 percent by volume. A nozzle is installed along the tank's central axis at one end of the tank to provide the capability to mix the fluid. Two heaters, designated as heater $\mathrm{A}$ and heater $\mathrm{B}$, are immersed in the fluid. Heater A is located at the top of the dome opposite the nozzle. Heater B is positioned off the tank's central axis, approximately $0.025 \mathrm{~m}$ away from the tank wall. The heaters are constructed of an etched-foil element which is encased in silicon rubber insulation and then sandwiched between two 304L stainless steel plates. The outside dimensions of the heater assembly are $0.1046 \mathrm{~m}$ by $0.0742 \mathrm{~m}$. Both heaters are the same size, except that heater $\mathrm{A}$ is bent to a $0.121-\mathrm{m}$ radius to follow the curvature of the tank wall. There is also a Liquid Acquisition Device (LAD) located at the bottom of the tank. The tank is made from a transparent acrylic to allow optical access. The geometry and dimensions of the TPCE tank are shown in Fig. 1.

The tank was initialized to a uniform temperature and pressure prior to the start of the jet mixing period. The initial values were set to $\mathrm{T}_{0}=296 \mathrm{~K}$ and $\mathrm{P}_{0}=41.164 \mathrm{kPa}$. The initial ullage location is approximated from the video of the experiment at the beginning of the jet mixing portion of each test run. The ullage is initialized as being spherical, but the CFD simulations are allowed to run for 5 seconds without any jet mixing in order to let the ullage shape reach an equilibrium state before the jet is activated. The initial ullage position and radius for each case are summarized in Table 2 below.

Table 2: Initial ullage position and radius

\begin{tabular}{cccc}
\hline Test Run & $\boldsymbol{X}, \boldsymbol{m}$ & $\boldsymbol{Y}, \boldsymbol{m}$ & Ullage Radius, $\boldsymbol{m}$ \\
\hline 11 & 0.005 & 0.26 & 0.0977 \\
15 & 0 & 0.26 & 0.0977 \\
13 & -0.003 & 0.26 & 0.0977 \\
4 & 0.015 & 0.19 & 0.1039 \\
\hline
\end{tabular}

In Table 2, $x$ is the distance from the tank's central axis (which passes through the nozzle) to the center of the ullage (the positive direction is towards the side heater) and $y$ is the distance from the bottom of the tank to the center of the ullage. The $z$ position of the center of the ullage was zero in every case, since it was not possible to determine it from the experimental video. All of the walls were modeled as adiabatic. The mass flow rate into the LAD was set equal to the mass flow rate out of the jet nozzle for each case. During the experiment, the residual acceleration was acting predominantly along the central axis of the tank. In the CFD simulations, a constant acceleration due to gravity of $1.0 \mathrm{e}-06 \mathrm{~g}$ was applied along the central tank axis in the negative $y$ direction.

The computational mesh that was used for the simulations is shown in Fig. 2. It consists of 1,505,726 polyhedral cells. This mesh is refined near the jet and near the expected ullage location. The simulations were performed using version 16 of the ANSYS Fluent CFD code. The full 3D geometry was modeled. The Second Order

American Institute of Aeronautics and Astronautics 
Upwind scheme was used to discretize the turbulence, energy, and momentum equations (cell values). The PISO scheme was used for the pressure-velocity coupling (cell values). The Least Squares Cell Based scheme was used for the gradient calculations (face values). The PRESTO! scheme was used for the pressure interpolation (face values). The Point Implicit (Gauss-Seidel) linear equation solver with the Algebraic Multi-Grid (AMG) method was used to solve the linearized systems of equations. The First Order Implicit temporal discretization scheme was used with the explicit VOF model with a time step size equal to 0.005 seconds. The Geometric Reconstruction scheme was used to discretize the VOF equation. The convergence criteria are set to $1 \times 10^{-4}$ for all of the equations except the energy equation, for which it is set to $1 \times 10^{-6}$.

\section{Results and Discussion}

The computational cases presented in this paper are based on the TPCE jet mixing tests that were conducted in microgravity ${ }^{1}$. The four different cases summarized in Table 1 above were simulated. Cases 11, 13 and 15 differ in their jet Weber numbers, while cases 15 and 4 have the same jet Weber number, but different initial ullage positions.

A grid independence study was conducted for a case with an ullage in zero gravity. The case was initialized with a spherical ullage shape and zero velocity in the tank. Due to the absence of a gravitational force acting on the tank, the velocity magnitude is expected to remain at zero, and the ullage shape is expected to remain spherical. However, the diffuse nature of the VOF model is prone to producing spurious velocities at the liquid-vapor interface. The goal of the grid independence study is to determine which grid will result in minimal spurious velocities and a minimally diffuse interfacial region. Three different grids were created. The results from these grids are compared in Figures 3 and 4 for the phase distribution and velocity vectors in the tank, respectively. The medium grid results in a reasonably sharp interface, which is similar to the one produced by the fine grid. It also predicts lower spurious velocities near the interface compared to the results of the fine grid. Based on this, the medium grid was used for the TPCE simulations.

Results from the four simulation case studies are compared with still images extracted from the experimental video data in Figures 5 - 18. Here, the CFD results are shown on the left, and the experimental image is shown on the right. The first CFD image on the left shows the front view of the tank, with the side heater (heater B) positioned on the right, heater A positioned at the top, and the jet nozzle and LAD located at the bottom of the image. Here, all of the walls inside the tank are shown as transparent grey 3D surfaces. The liquid-ullage interface is shown as a transparent yellow 3D surface. The tank center plane is shown as an opaque surface colored by the velocity magnitude to indicate the jet location and spread; flow streamlines are also displayed on this plane. The second CFD image on the left is the top view of the tank; this corresponds to a cross-sectional plane which is also displayed on the front view. This cross-sectional plane is also colored by the velocity magnitude, and again features flow streamlines. The timing between the experimental video images is not synchronized with the timing of the CFD images. As a result, the comparison is qualitative in nature, and its purpose is to show that the CFD model predicts features of the ullage dynamics that are similar to the ones observed in the experiment.

Figure 5 shows a comparison between the experimental results and the CFD predictions after 55.7 seconds of mixing for TPCE case 11 (see Table 1). Since this test uses a low flow rate, the jet does not penetrate or break the ullage, but it does create a small indentation at the bottom of the interface. Since the ullage shape and the depth of the indentation remain unchanged after a few seconds from the initiation of jet mixing, the CFD simulation was only performed for the first 60 seconds of mixing. As shown in Fig. 5, the predicted shape and position of the ullage are very similar to the experimental ones. The predicted depth of the indentation created by the jet at the interface is also very close to the experimental one. The CFD results show that although the ullage shape is almost axisymmetric, the flow at the tank center plane is not axisymmetric. This is mainly due to the asymmetry in the tank geometry created by the side heater.

The CFD results for TPCE case 15, which has a higher flow rate than case 11 (see Table 1), but a similar initial ullage position, are compared with the video from the experiment at 9, 54, 115 and 244 seconds of mixing in Fig. 6 - 9. In this case, the axial jet initially pierces through the ullage (Fig. 6), and then pushes it against the top heater (Fig. 7-9). The ullage stays near the top of the tank while it is fully penetrated by the jet. After the ullage is pushed against the top heater, it oscillates slightly from left to right in both the CFD model and the experiment, as shown in Fig. 7 and 8. Here, the predicted shape and position of the ullage are very similar to the experimental ones. However, after 4 minutes of mixing, as shown in Fig. 9, the ullage moves asymmetrically to the side of the tank opposite the location of the side heater in the experiment, but in the CFD model, the extent of the asymmetric repositioning is noticeably smaller. This could be due to possible differences in the nozzle or top heater alignments between the experiment and the simulations.

American Institute of Aeronautics and Astronautics 
TPCE case 13 has the highest jet flow rate of all of the tests considered in this study. In this case, the jet pierces through and breaks the ullage. The results of the simulation are compared with the experiment at $6,9,51,124$ and 130 seconds of mixing in Fig. 10 - 14. Initially, the jet pierces through the center of the ullage, as shown in Fig. 10. The flow at the center plane of the tank, as indicated by the streamlines shown in Fig. 10, is nearly symmetric. The CFD predictions are very similar to the experiment. As mixing progresses, an elongated ullage shape which resembles an apple core is observed in both the CFD model and the experiment, as indicated in Fig. 11. At this time, the flow at the center plane of the tank remains nearly symmetrical, and the penetration location stays at the center of the ullage. After about a minute of mixing, the jet breaks the ullage, creating a partial torus shape, and moves the ullage to the side of the tank opposite heater B as shown in Fig. 12. Here, significant asymmetry in the flow and in the ullage shape can be observed. The CFD model and the experiment show very similar ullage shapes and positions, except that in the experiment, a small vapor bubble remains attached to the tank wall right above heater B. In Fig 13, as mixing progresses, the ullage has rotated around the central axis of the tank from its position in Fig. 12; it is also pushed against the front wall of the tank. The ullage also becomes elongated and extends towards the nozzle. The shape of the ullage in the CFD model is similar to that of the experiment, as evident in Fig. 13. Figure 14 shows that after about 2 minutes of mixing, the jet pushes the ullage against the top heater, and pierces through its center. The ullage maintains a partial torus shape, as can be seen in the top view included in Fig. 14. The predicted ullage shape shown in Fig. 14 is very similar to its experimental counterpart; however, it is hard to discern from the experimental image whether the ullage has a full or partial torus shape.

TPCE case 4 has the same jet flow rate as case 15 (see Table 1); however, in the beginning of the jet mixing period, the ullage is located closer to the nozzle at the center of the tank. In this case, the jet initially pierces through the ullage, and then pushes it towards the top heater. Later in the simulation, the ullage stays near the top of the tank while it is fully penetrated by the jet. This behavior is very similar to that observed in case 15 . The results of the simulation are compared with the experiment at 1.5, 1.9, 35 and 73 seconds of mixing in Fig. 15-18, respectively.

The simulation results presented here using the VOF model of the ANSYS Fluent CFD code have successfully reproduced the main features of the jet-ullage interaction for three different jet flow rates and two different initial ullage positions. The focus of the current study was on modeling the fluid flow and interface dynamics. While the thermal effects of the heaters and the interfacial mass transfer were neglected here, they will be examined in future work.

\section{Conclusion}

A two-phase compressible VOF CFD model was utilized for modeling fluid flow and ullage dynamics during the jet mixing portion of the Tank Pressure Control Experiment ${ }^{1}$ (TPCE). Three different jet Weber numbers and two different initial ullage positions were considered. Three of the modeled cases, TPCE case 11 with jet We $=0.71$, TPCE case 15 with jet $\mathrm{We}=4.74$, and TPCE case 13 with jet $\mathrm{We}=15.55$, have initial ullage positions near the top heater (heater A). The fourth case that was modeled, TPCE case 4, has the same jet Weber number as TPCE case 15, but the initial ullage location is at the center of the tank, closer to the jet nozzle. In the case with the lowest Weber number studied, the jet did not fully penetrate the ullage, creating an indentation at the interface. In the cases with higher Weber numbers, the jet penetrated the ullage, forming a torus shape, and pushed it towards the heater opposite the nozzle. In the case with the highest Weber number studied, the jet initially pierced through the ullage, and later broke it, resulting in a partial torus shape. In the case where the ullage was initially located in the middle of the tank closer to the nozzle, the jet pierced through the ullage and impinged on the heater surface before pulling the ullage towards the heater. In all four cases, the CFD model was able to capture the ullage dynamics with good qualitative fidelity. The focus of the present study was on modeling the fluid dynamics and jet-ullage interaction. The thermal effects of the heaters, and the impact of the interfacial mass transfer, will be examined in future work in order to study their potential effects on jet-ullage interactions and on the tank pressure evolution.

\section{Acknowledgments}

This work was supported by the NASA Space Technology Mission Directorate's Technology Demonstration Missions Program under the Evolvable Cryogenics Project.

\section{References}

${ }^{1}$ Bentz, M. D., Tank Pressure Control in Low Gravity by Jet Mixing, NASA-CR-191012, March, 1993

${ }^{2}$ Wendl, M. C., Hochstein, J.I. and Sasmal, G.P. "Modeling of Jet-Induced Geyser Formation in a Reduced Gravity Environment," AIAA-1991-803, 1991

American Institute of Aeronautics and Astronautics 
${ }^{3}$ Breisacher K. and Moder, J. "Preliminary Simulations of the Ullage Dynamics in Microgravity during the Jet Mixing Portion of the Tank Pressure Control Experiments," AIAA 2015-3853, AIAA 50 ${ }^{\text {th }}$ Joint Propulsion Conference, AIAA, Orlando, FL, 2015

${ }^{4}$ Lemmon, E.W., McLinden, M.O., and Friend, D.G., "Thermophysical Properties of Fluid Systems" in NIST Chemistry WebBook, NIST Standard Reference Database Number 69, Eds. Linstrom, P.J., and Mallard, W.G., National Institute of Standards and Technology, Gaithersburg MD, 20899, http://webbook.nist.gov.

${ }^{5}$ Hirt, C.W., and Nichols B.D., "Volume of fluid (VOF) method for the dynamics of free boundaries," Journal of Computational Physics, Vol. 39 No. 1, 1981, pp. 201-225.

${ }^{6}$ Brackbill J.U., Kothe, D.B., Zemach, C., “A continuum method for modeling surface tension,” J. Comp. Phys. Vol. 100, 1992, pp. 335-354.

${ }^{7}$ Menter, F. R., "Two-Equation Eddy-Viscosity Turbulence Models for Engineering Applications,” AIAA Journal, Vol. 32 No. 8, 1994, pp. 1598-1605

${ }^{8}$ Wilcox, D.C., Turbulent Modeling for CFD, DCW Industries, Inc., La Canada, California, 1998

${ }^{9}$ Bradshaw, P., Ferriss, D.H., and Atwell, N.P., "Calculation of Boundary-Layer Development Using the Turbulent Energy Equation," Journal of Fluid Mechanics, Vol. 28, No. 3, 1967, pp. 593-616.

${ }^{10}$ ANSYS Fluent Documentation. Release 16.0. November 2014.

American Institute of Aeronautics and Astronautics 


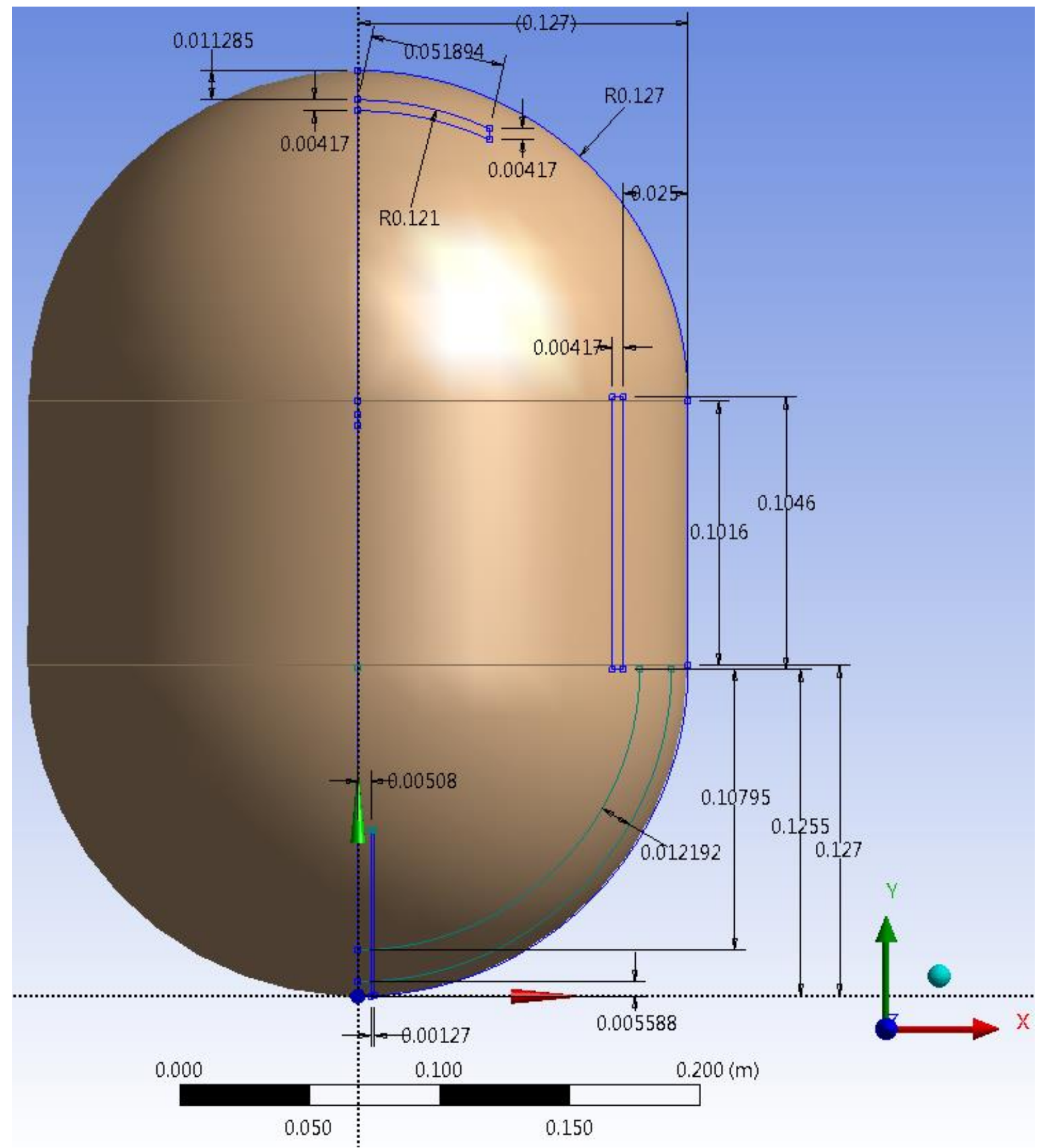

Figure 1: TPCE tank geometry and dimensions 


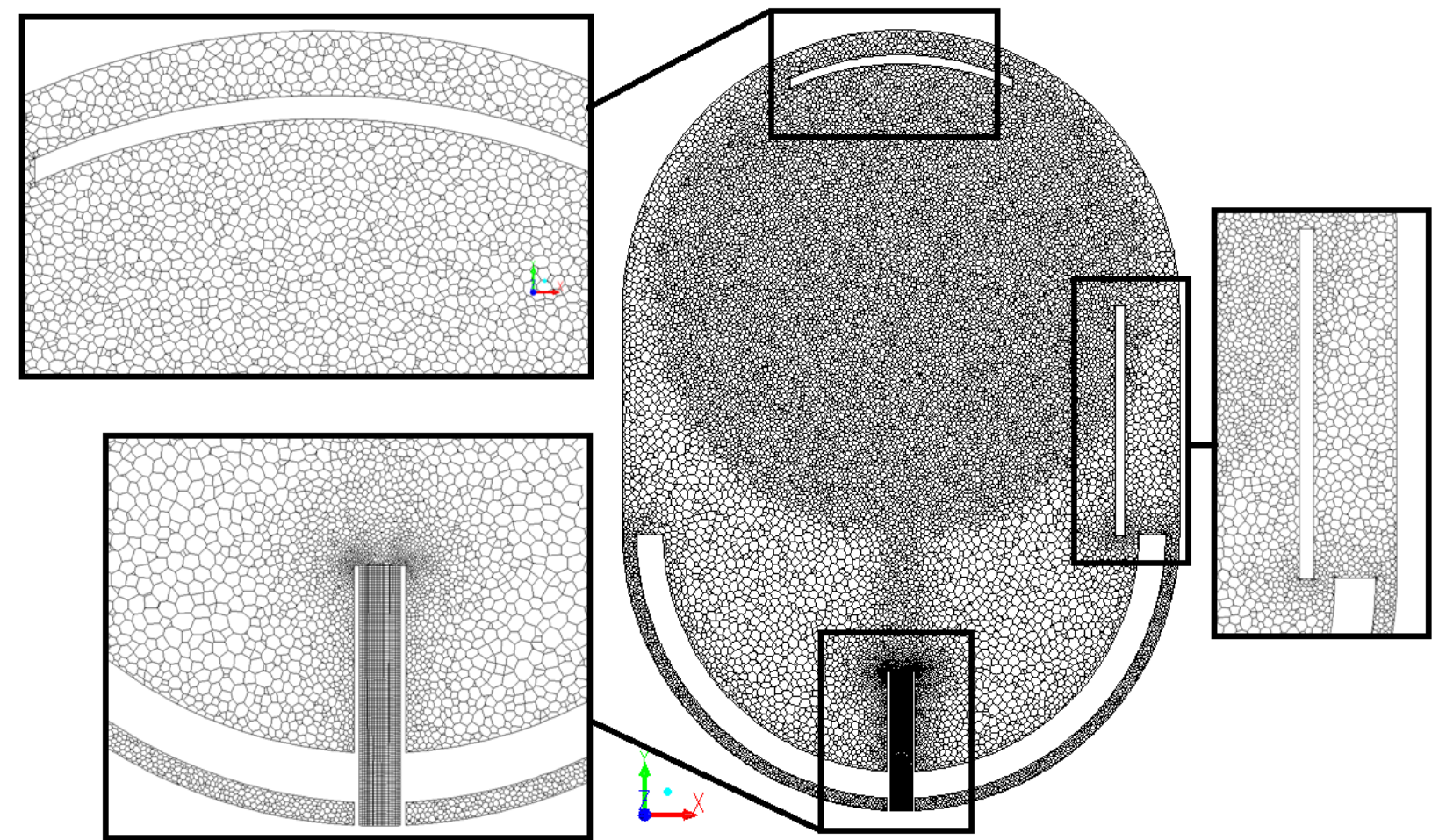

Figure 2: 3D computational mesh used for modeling TPCE jet mixing cases (1,505,726 polyhedral cells)

\section{Volume fraction of gas}
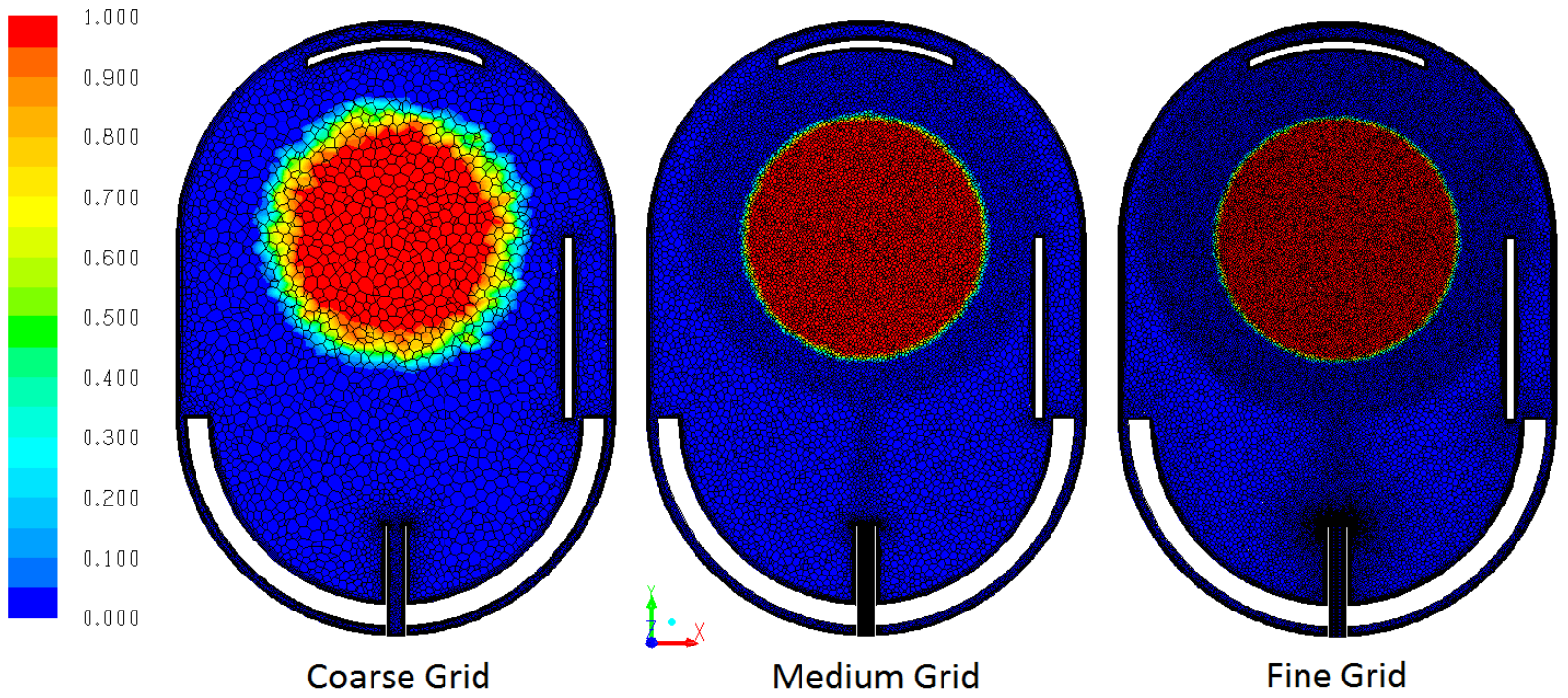

Figure 3: Grid Independence Study: Phase distribution in the tank compared between different grids (Coarse Grid 441,086 cells; Medium Grid - 1,505,726 cells; Fine Grid - 4,455,339 cells) 


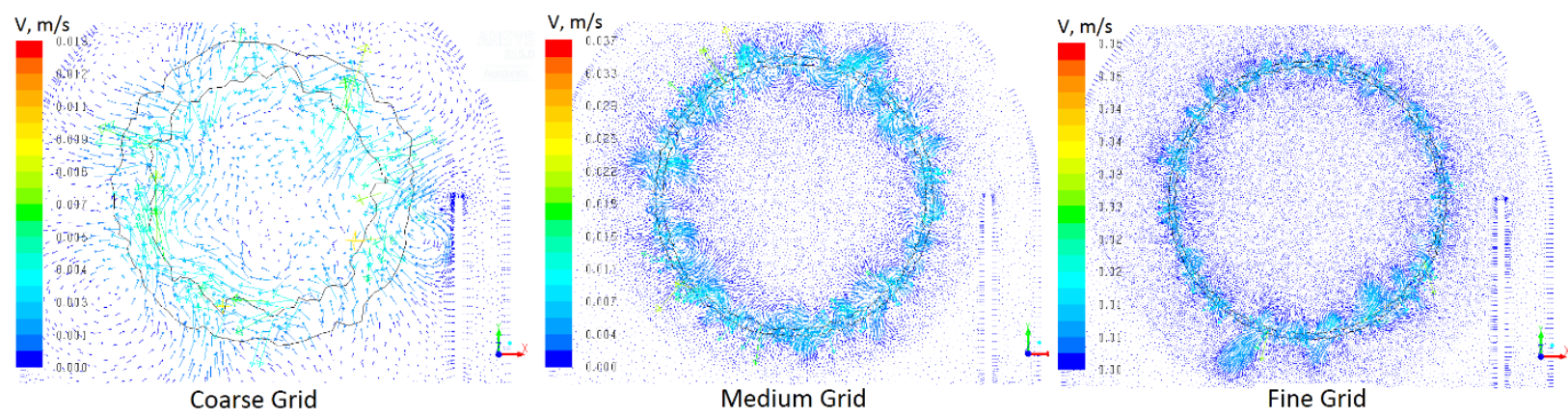

Figure 4: Grid Independence Study: Velocity vectors near the interface compared between different grids (Coarse Grid - 441,086 cells; Medium Grid - 1,505,726 cells; Fine Grid - 4,455,339 cells)
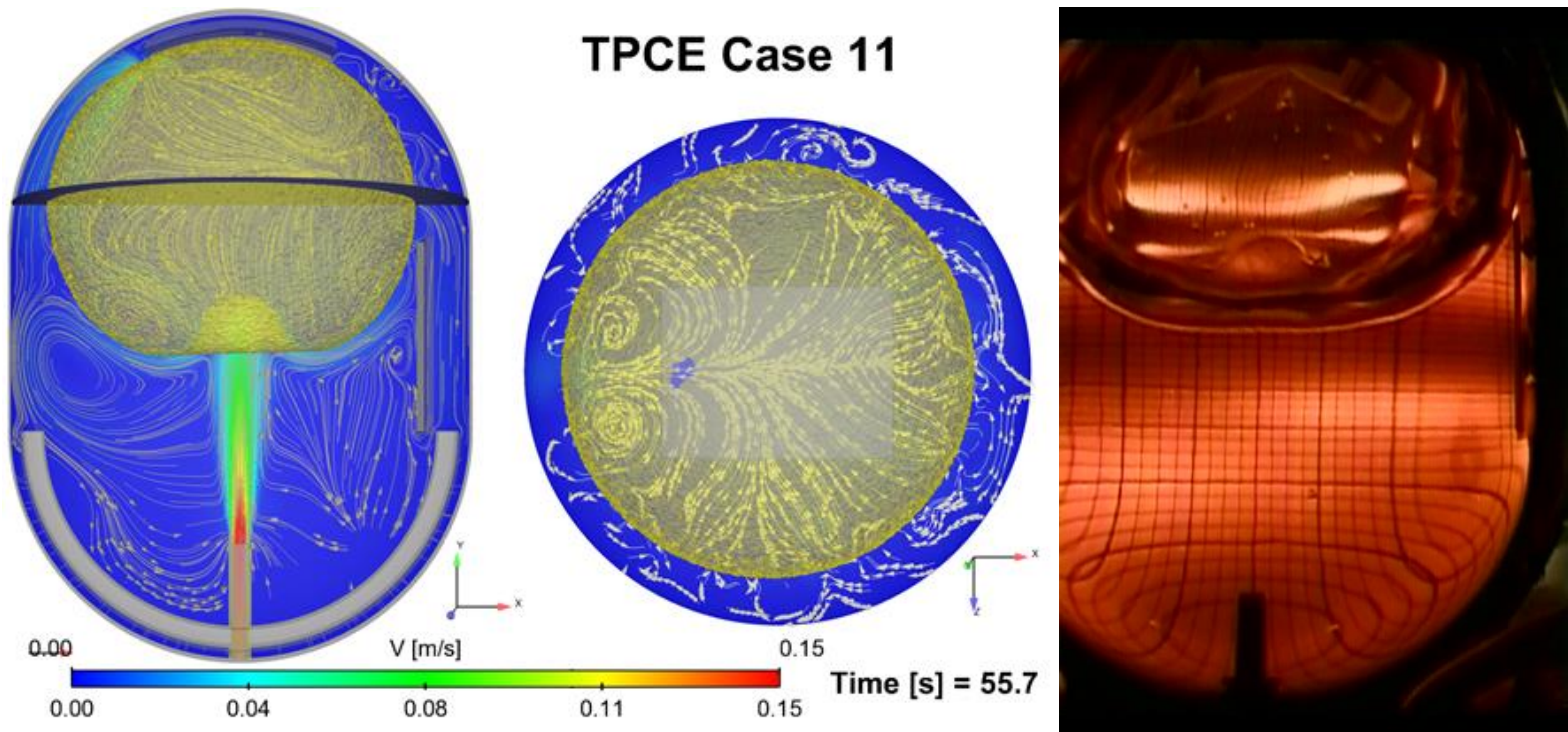

Figure 5: TPCE Case 11: tank at 55.7 seconds of mixing: experiment (right); CFD 3D ullage and center plane colored by velocity magnitude with streamlines (front view - left, top view - right)
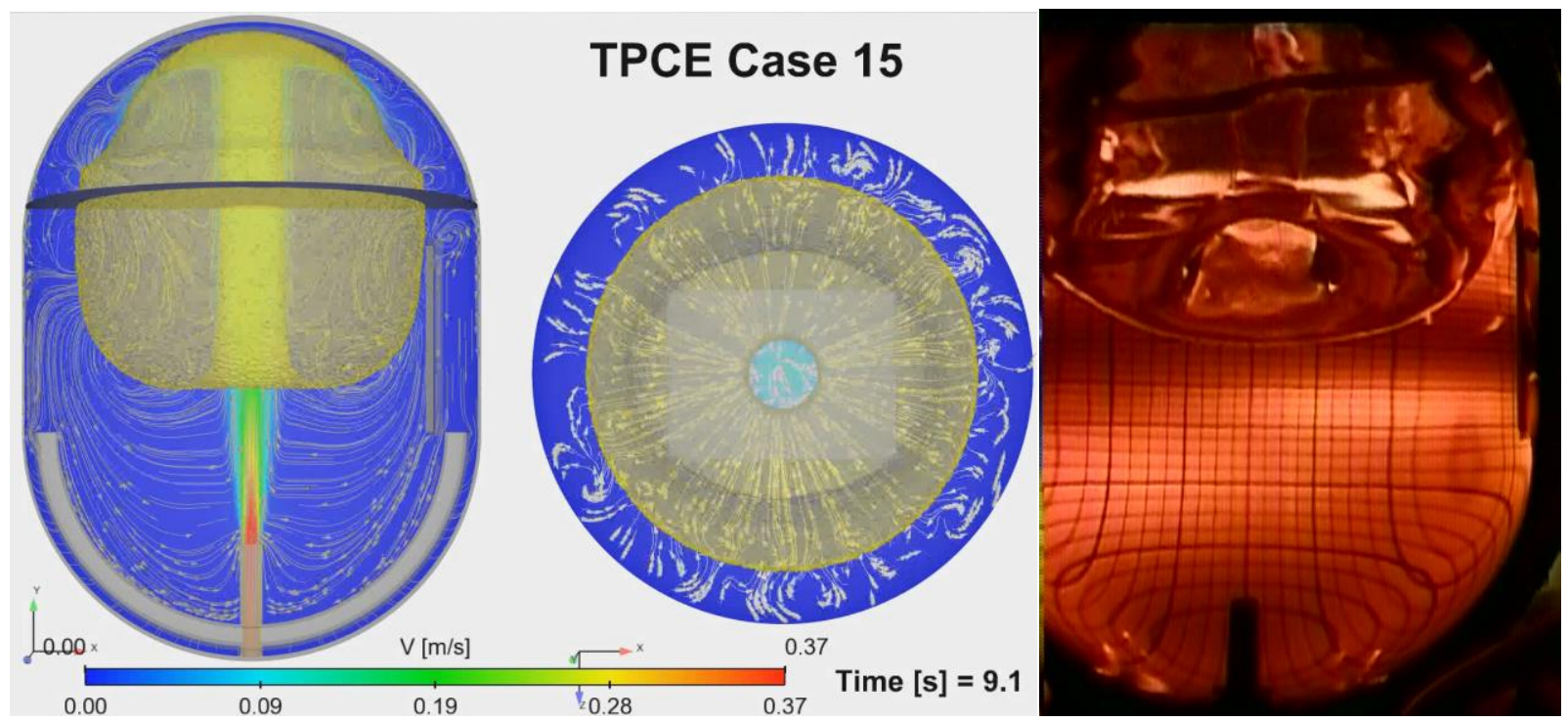

Figure 6: TPCE Case 15: tank at 9 seconds of mixing: experiment (right); CFD 3D ullage and center plane colored by velocity magnitude with streamlines (front view - left, top view - right)

American Institute of Aeronautics and Astronautics 

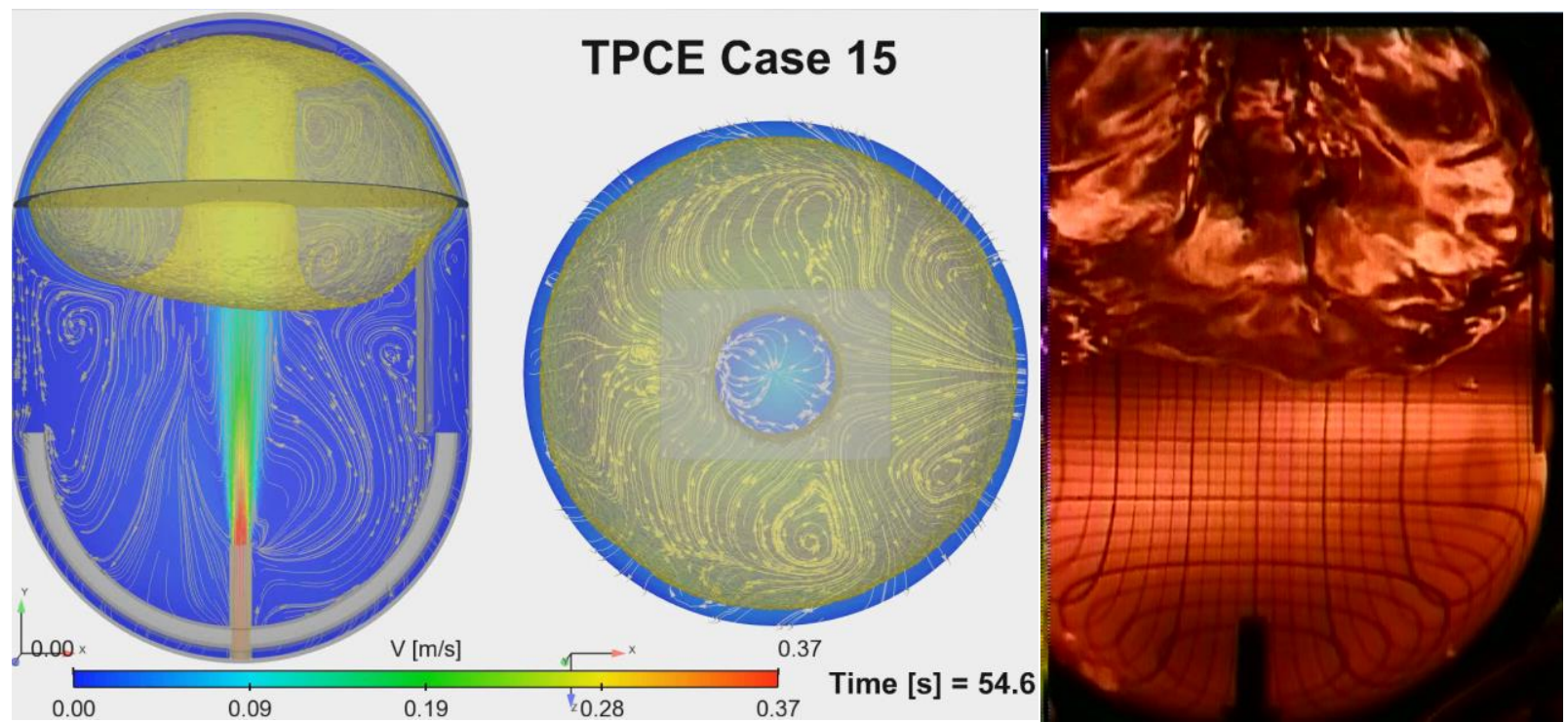

Figure 7: TPCE Case 15: tank at 54 seconds of mixing: experiment (right); CFD 3D ullage and center plane colored by velocity magnitude with streamlines (front view - left, top view - right)

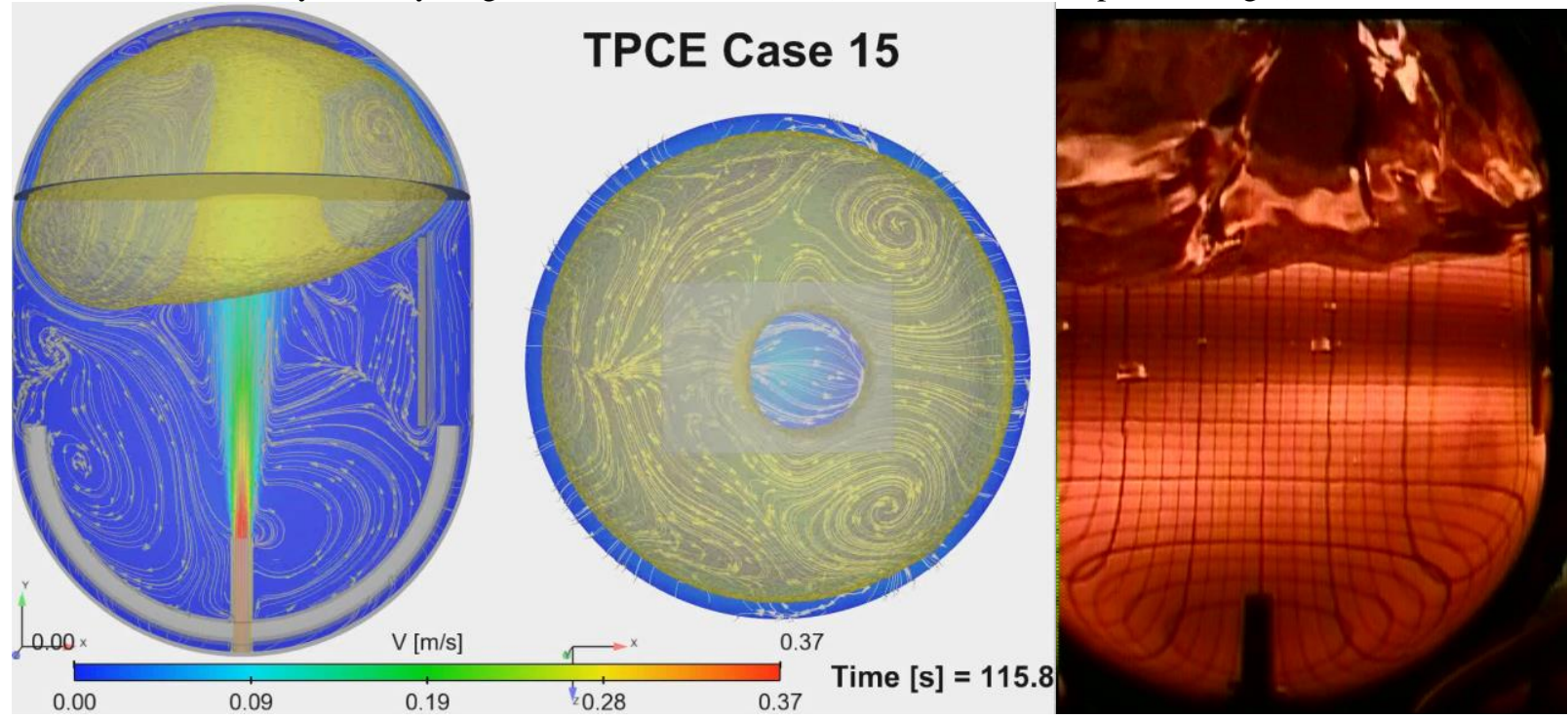

Figure 8: TPCE Case 15: tank at 115 seconds of mixing: experiment (right); CFD 3D ullage and center plane colored by velocity magnitude with streamlines (front view - left, top view - right) 


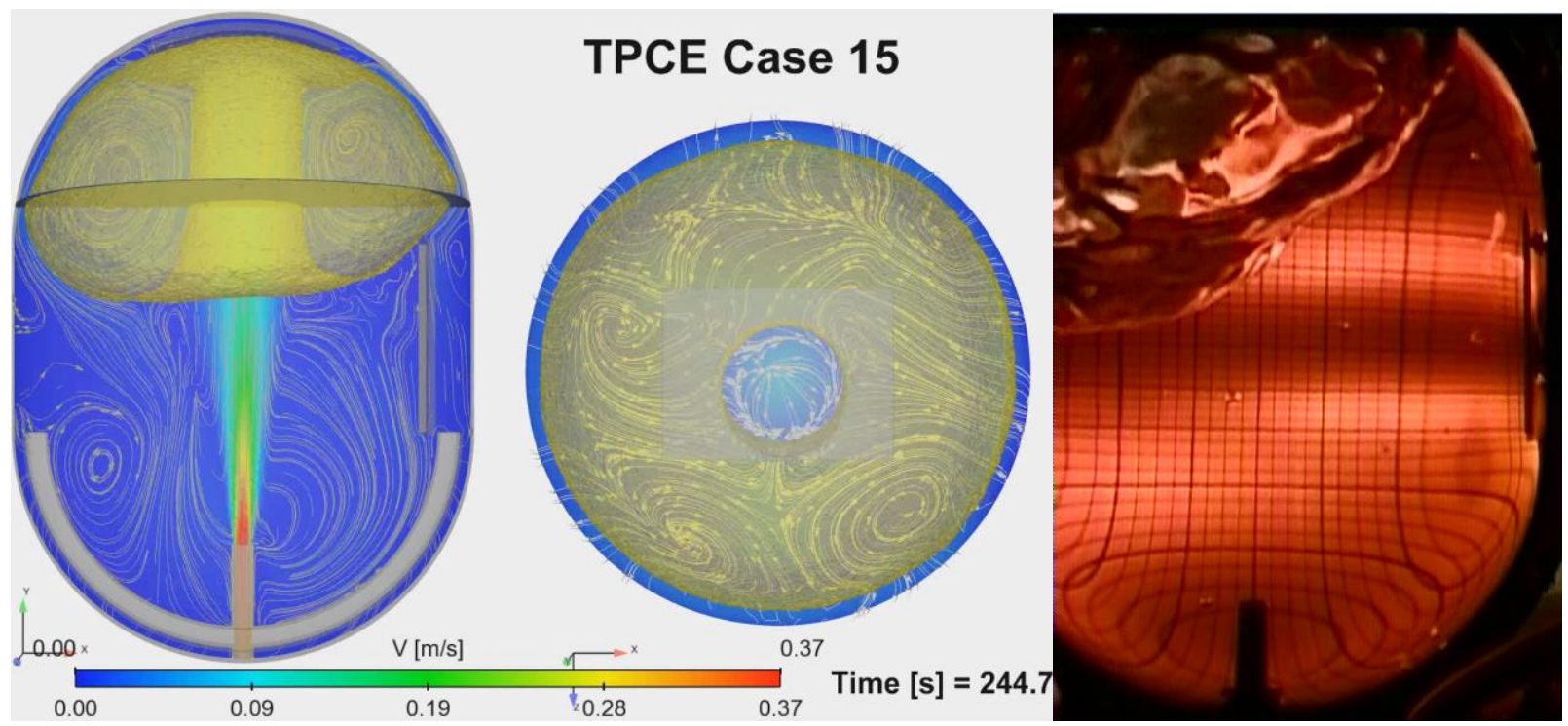

Figure 9: TPCE Case 15: tank at 244 seconds of mixing: experiment (right); CFD 3D ullage and center plane colored by velocity magnitude with streamlines (front view - left, top view - right)

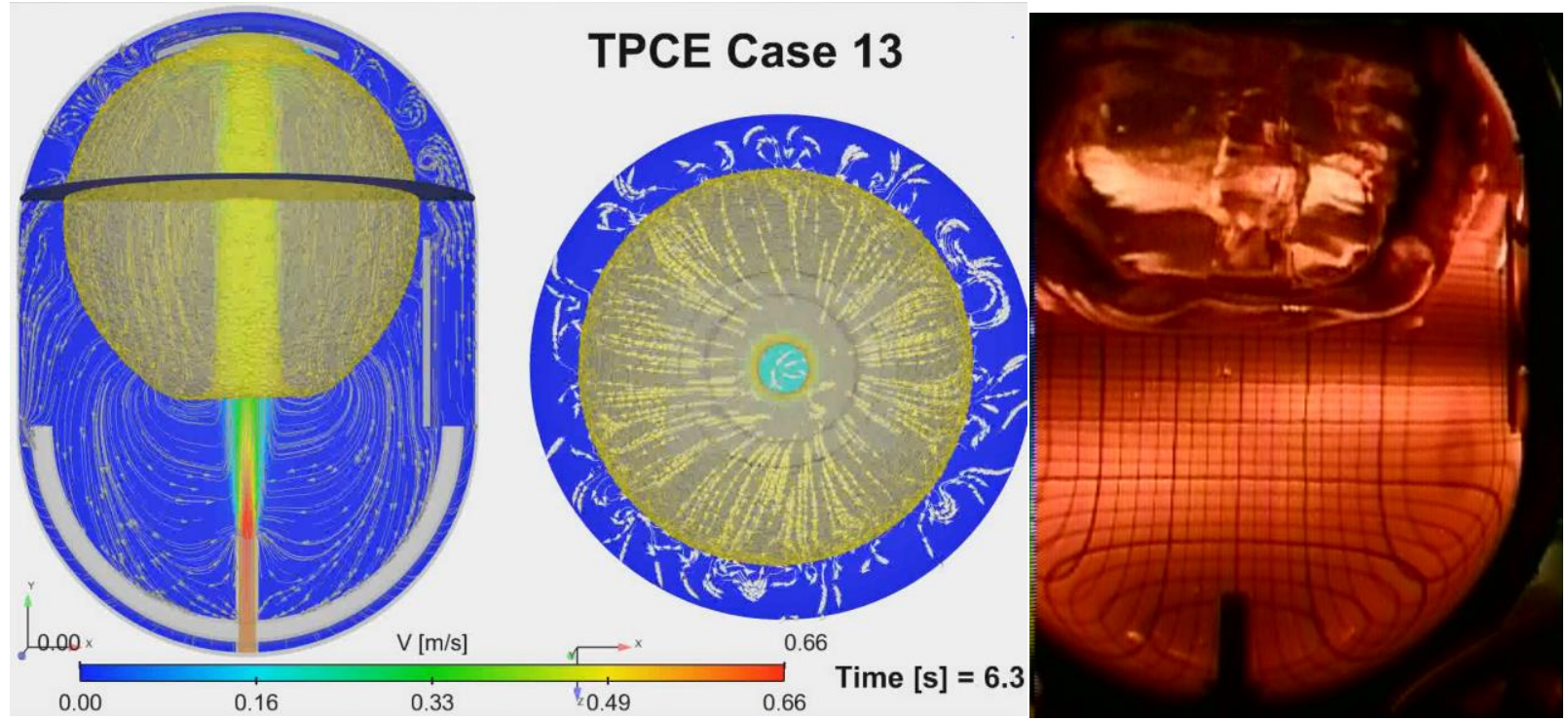

Figure 10: TPCE Case 13: tank at 6 seconds of mixing: experiment (right); CFD 3D ullage and center plane colored by velocity magnitude with streamlines (front view - left, top view - right) 

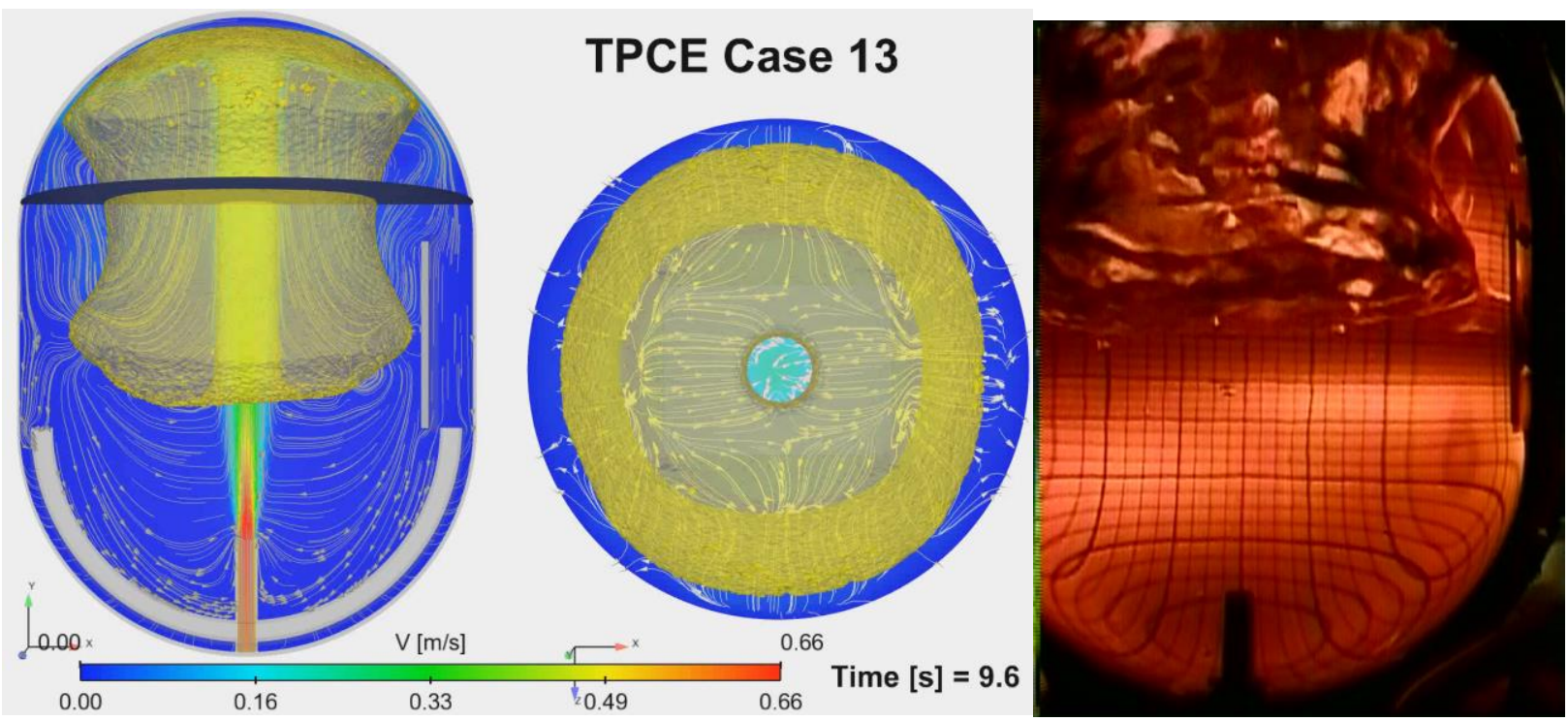

Figure 11: TPCE Case 13: tank at 9 seconds of mixing: experiment (right); CFD 3D ullage and center plane colored by velocity magnitude with streamlines (front view - left, top view - right)

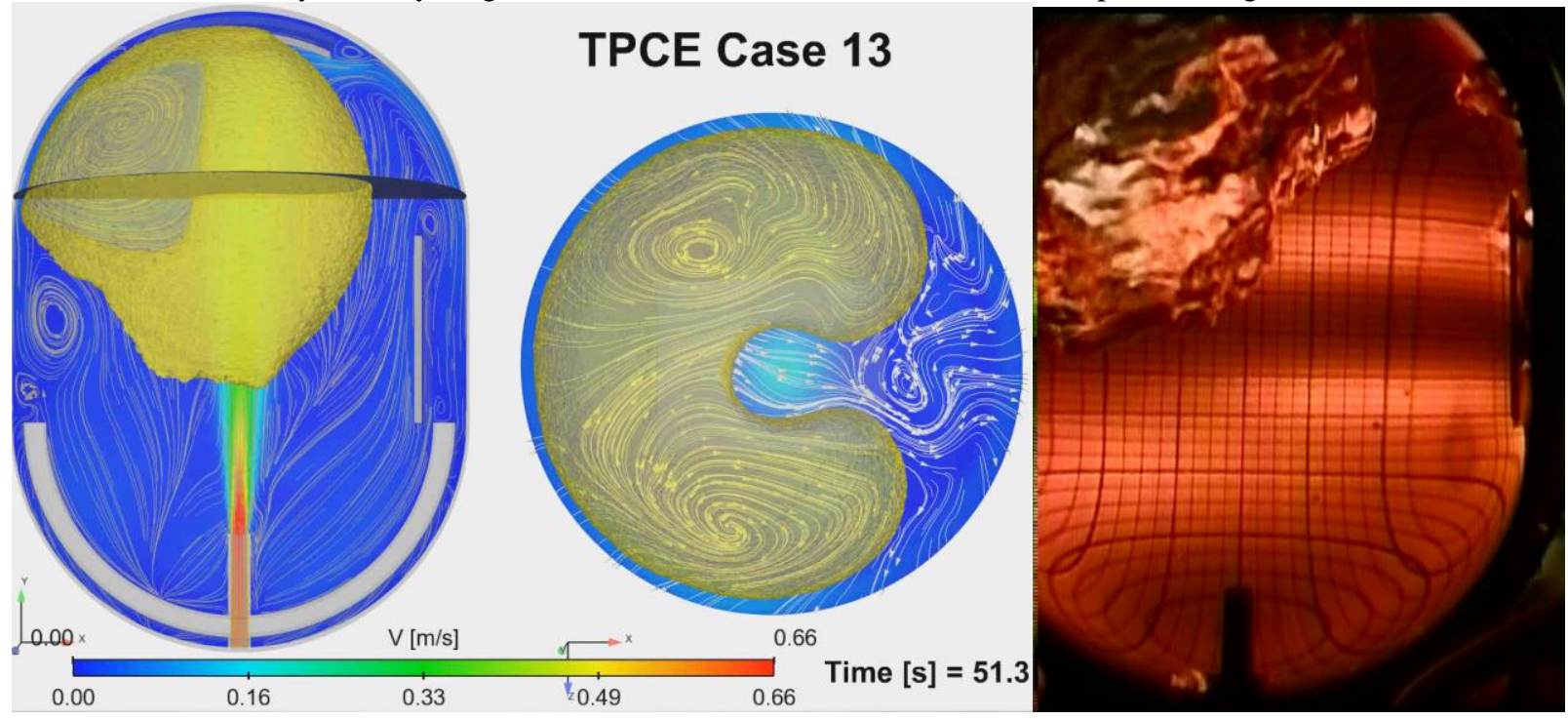

Figure 12: TPCE Case 13: tank at 51 seconds of mixing: experiment (right); CFD 3D ullage and center plane colored by velocity magnitude with streamlines (front view - left, top view - right) 


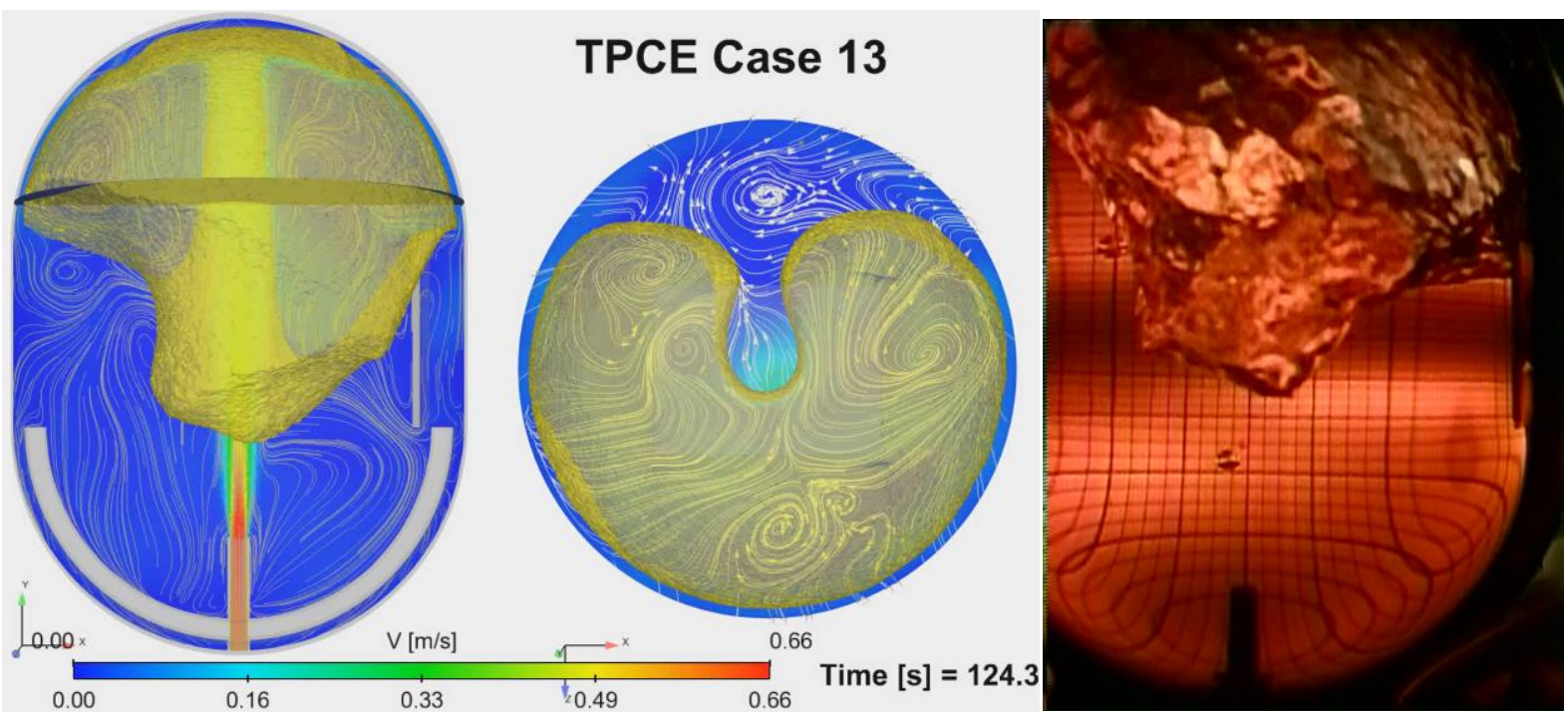

Figure 13: TPCE Case 13: tank at 124 seconds of mixing: experiment (right); CFD 3D ullage and center plane colored by velocity magnitude with streamlines (front view - left, top view - right)

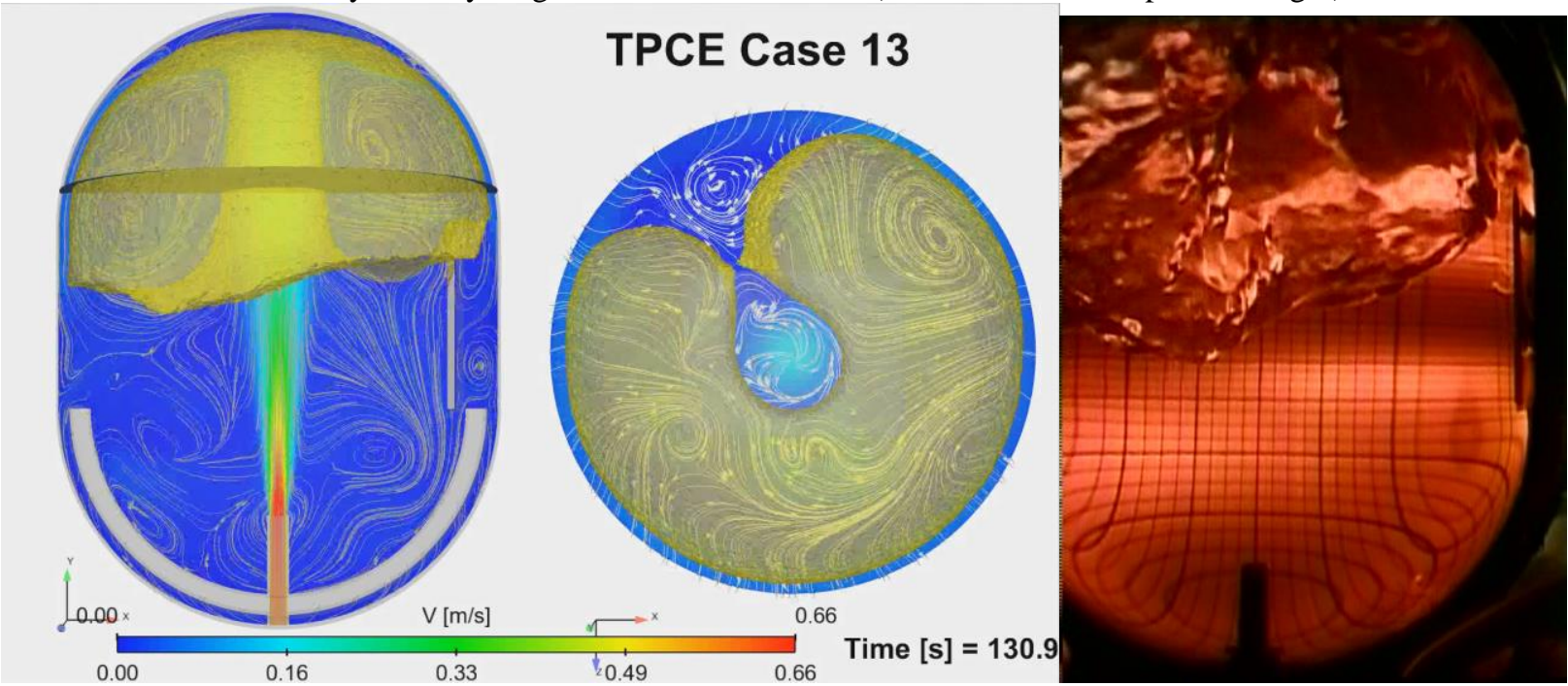

Figure 14: TPCE Case 13: tank at 130 seconds of mixing: experiment (right); CFD 3D ullage and center plane colored by velocity magnitude with streamlines (front view - left, top view - right) 

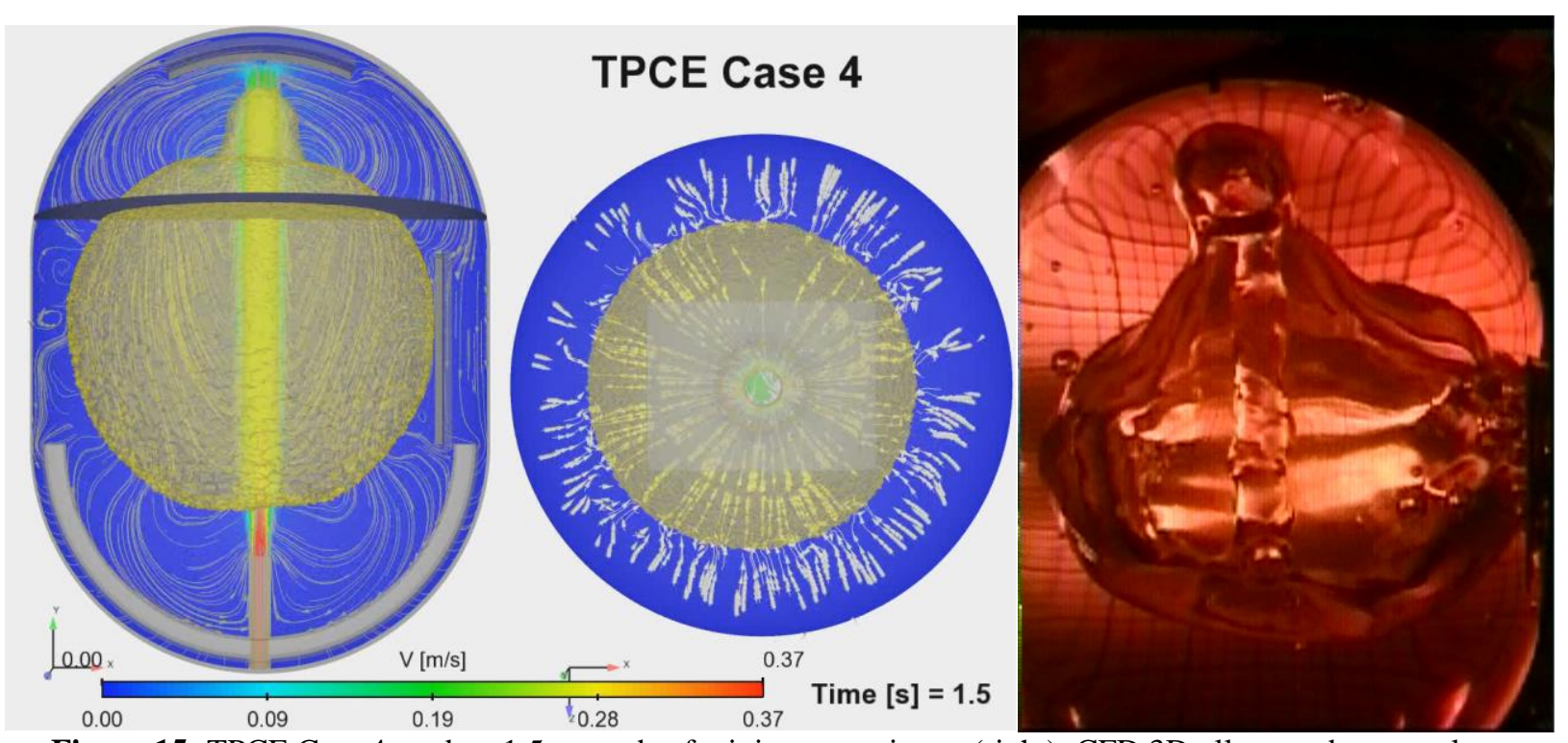

Figure 15: TPCE Case 4: tank at 1.5 seconds of mixing: experiment (right); CFD 3D ullage and center plane colored by velocity magnitude with streamlines (front view - left, top view - right)
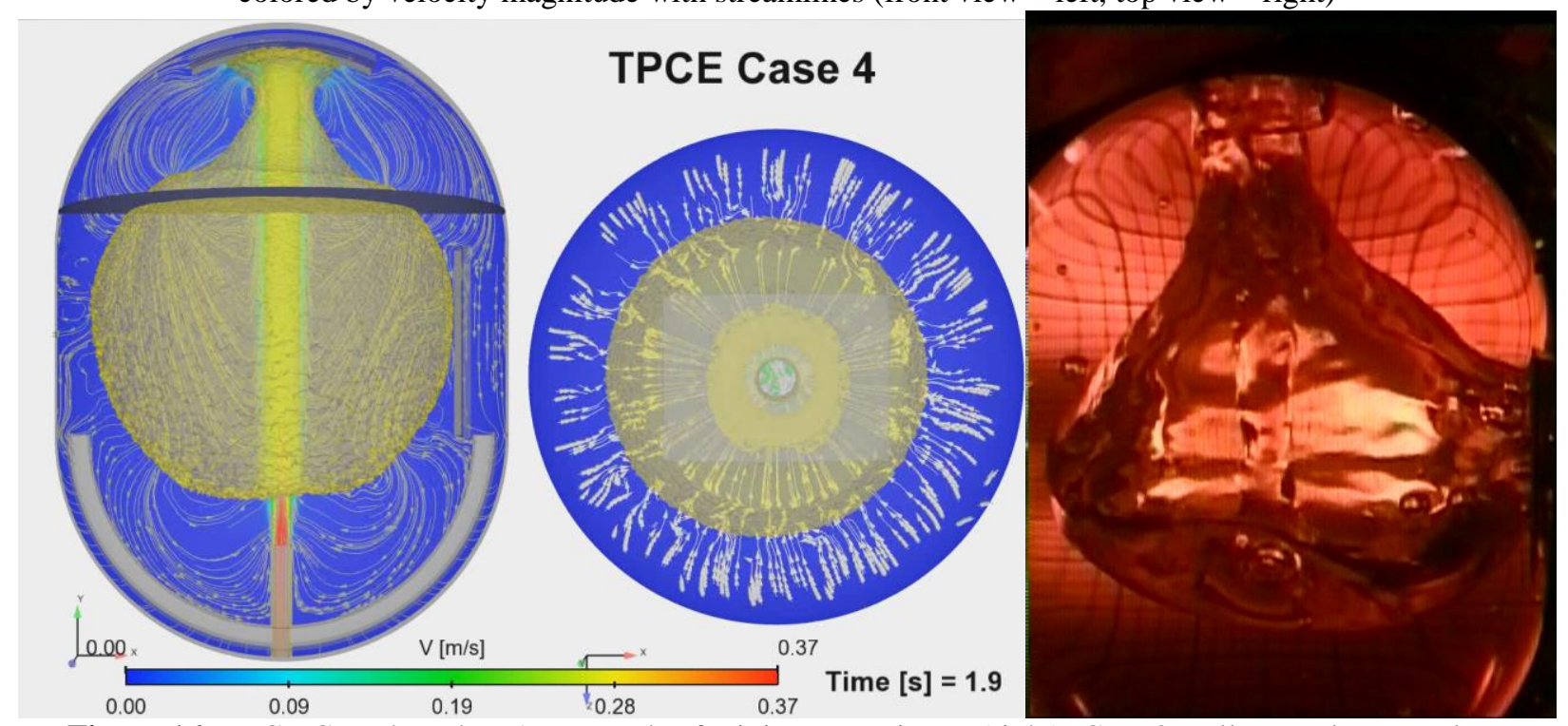

Figure 16: TPCE Case 4: tank at 1.9 seconds of mixing: experiment (right); CFD 3D ullage and center plane colored by velocity magnitude with streamlines (front view - left, top view - right) 

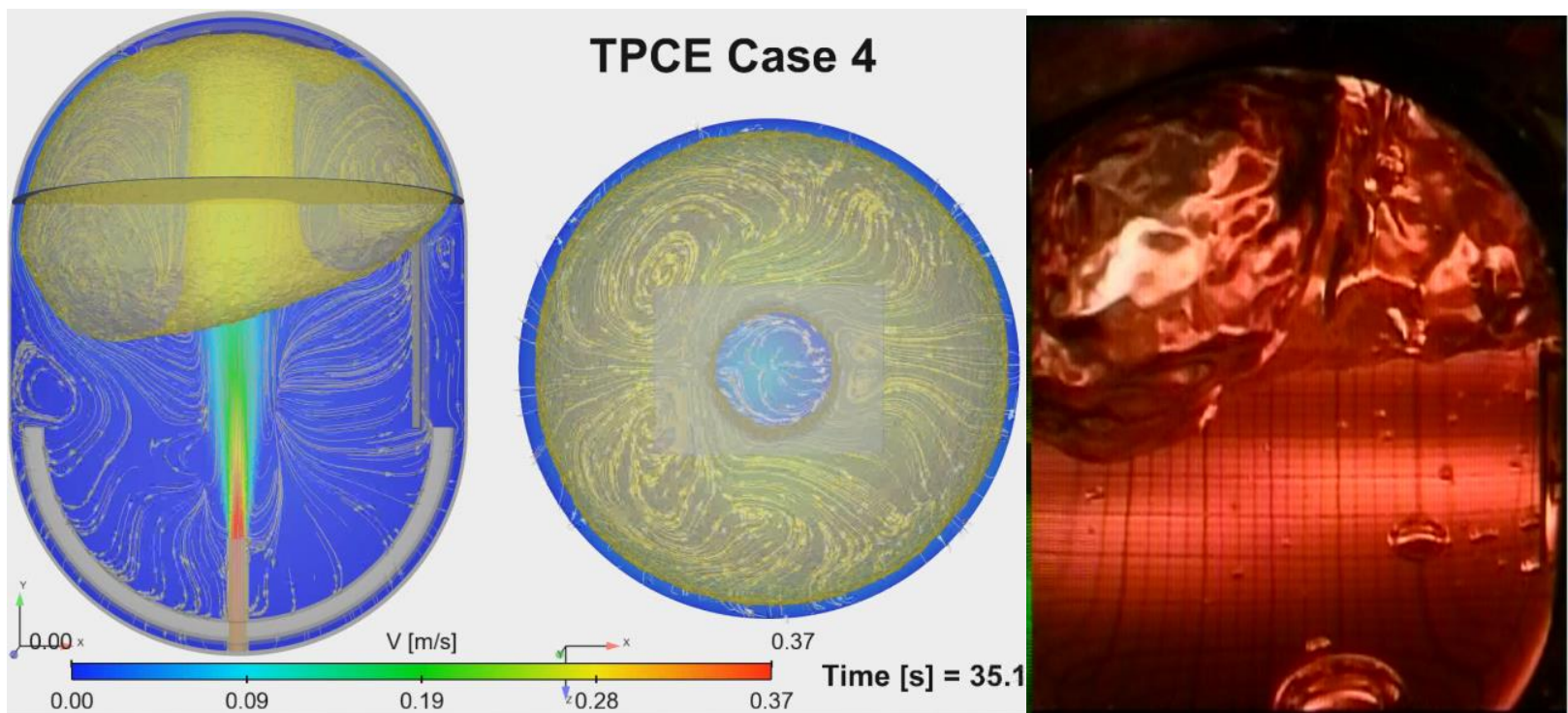

Figure 17: TPCE Case 4: tank at 35 seconds of mixing: experiment (right); CFD 3D ullage and center plane colored by velocity magnitude with streamlines (front view - left, top view - right)
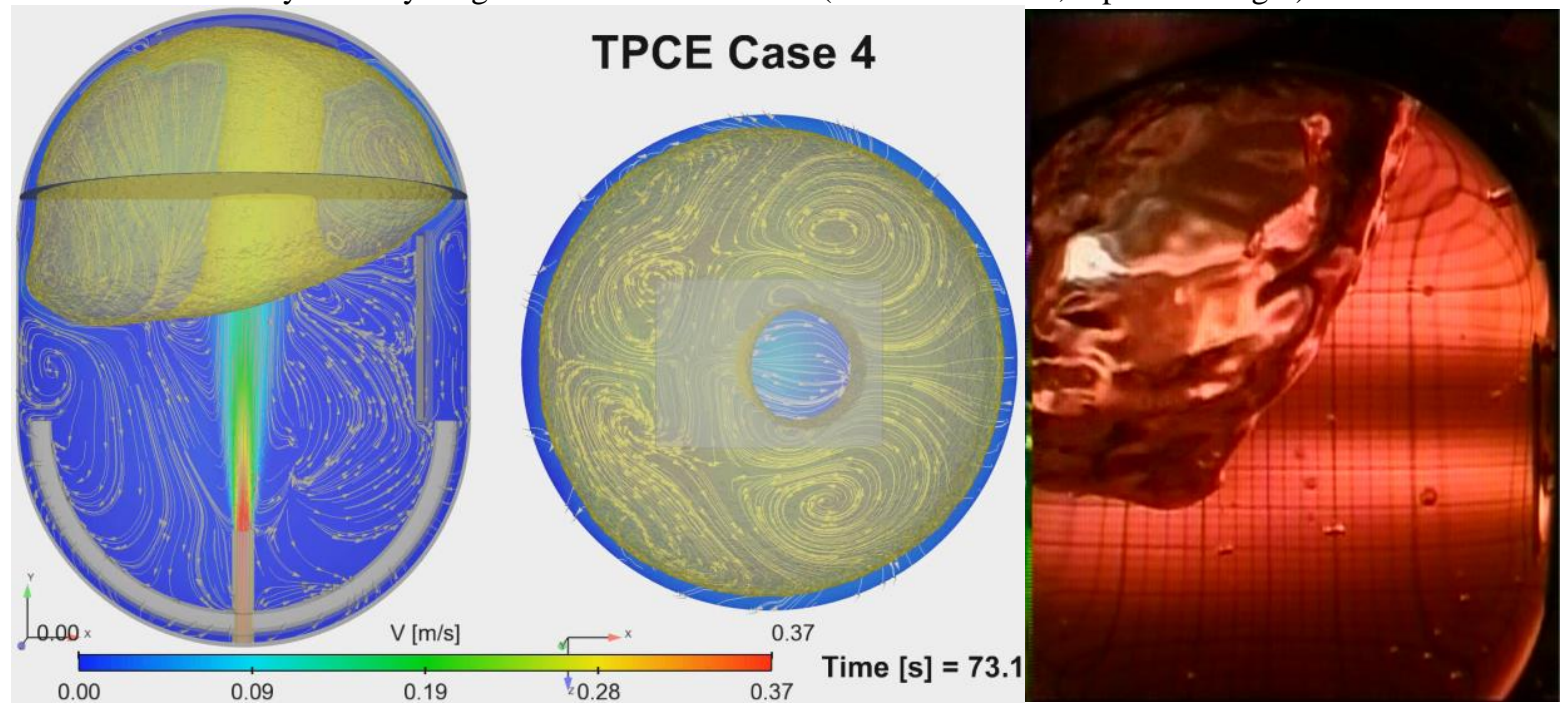

Figure 18: TPCE Case 4: tank at 73 seconds of mixing: experiment (right); CFD 3D ullage and center plane colored by velocity magnitude with streamlines (front view - left, top view - right) 